\title{
The 4 Year COBE DMR data is non-Gaussian
}

\author{
Pedro G. Ferreira ${ }^{1,2}$, Krzysztof M. Górski ${ }^{3,4}$, João Magueijo $^{5}$ \\ 1 Theory Group, CERN, CH-1211, Geneve 23 ,Switzerland \\ ${ }^{2}$ CENTRA, Instituto Superior Técnico, Lisboa 1096 Codex, Portugal \\ ${ }^{3}$ Theoretical Astrophysics Center, Juliane Maries Vej 30, DK-2100, Copenhagen Ø, Denmark \\ ${ }^{4}$ Warsaw University Observatory, Warsaw, Poland \\ ${ }^{5}$ Theoretical Physics, Imperial College, Prince Consort Road, London SW7 2BZ, UK
}

\begin{abstract}
I review our recent claim that there is evidence of non-Gaussianity in the 4 Year COBE DMR data. I describe the statistic we apply, the result we obtain and make a detailed list of the systematics we have analysed. I finish with a qualitative understanding of what it might be and its implications.
\end{abstract}

\section{INTRODUCTION}

In a recent letter [1] we have claimed that the 4 Year COBE DMR data exhibits evidence of non Gaussianity; our quantitative claim was that the hypotheses that this data set is due to a Gaussian random process can be ruled out at the $98 \%$ confidence level. This came somewhat as a surprise given that this data set was lauded as strong evidence for the inflationary paradigm: its statistics were thought to be consistent with Gaussianity [2].

We have performed an extensive analysis of the 4 year COBE DMR data set and have been unable to find a non cosmological origin for the non-Gaussian signal. The resuls of this analysis are presented in [3]. In this report I will only address a few often raised questions:

- Why does this statistic get a different result from all the ones that were previously used?

- Isn't the effect we are seeing just some systematic effect of the analysis?

- If it is signal what could it be?

\section{THE STATISTIC AND RESULT}

In our analysis we propose, and work with, an estimator for the normalized bispectrum. To construct such an estimator we work in the spherical harmonic representation $a_{\ell m}$ and consider the tensor product of 3 $\Delta T_{\ell}=\sum_{m} a_{\ell m} \mathrm{~s}$ One is interested in rotationally invariant quantities. These can be trivially obtained if one rewrites the tensor product in terms of the total angular momentum basis. The coefficient of the singlet will be the higher order invariant we are looking for. This procedure leads to an estimator of the bispectrum

$$
\begin{aligned}
\hat{B}_{\ell} & =\alpha_{\ell} \sum_{m_{1} m_{2} m_{3}} \mathcal{W}_{m_{1} m_{2} m_{3}}^{\ell \ell \ell} a_{\ell m_{1}} a_{\ell m_{2}} a_{\ell m_{3}} \\
\alpha_{\ell} & =\frac{1}{(2 \ell+1)^{\frac{3}{2}}}\left(\mathcal{W}_{000}^{\ell \ell \ell}\right)^{-1}
\end{aligned}
$$

where $\mathcal{W}_{m_{1} m_{2} m_{3}}^{\ell_{1} \ell_{2} \ell_{3}}$ are the Wigner $3 \mathrm{~J}$ coefficients. $\hat{B}_{\ell}$ may then be divided by the appropriate power of an estimator for $C_{\ell}$ in order to make it dimensionless, and suitably normalised. 


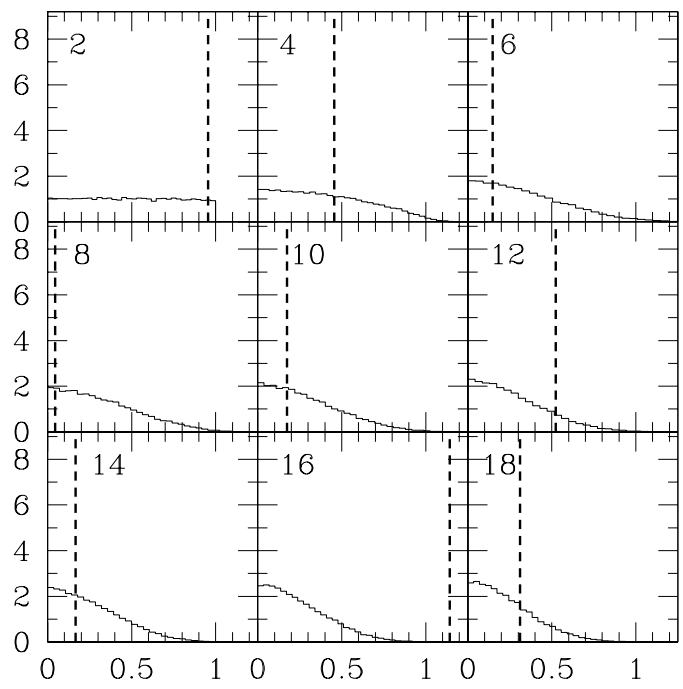

FIGURE 1. The vertical thick dashed line represents the value of the observed $I_{\ell}^{3}$. The solid line is the probability distribution function of $I_{\ell}^{3}$ for a Gaussian sky with extended galactic cut and DMR noise.

$$
I_{\ell}^{3}=\left|\frac{\hat{B}_{\ell}}{\left(\hat{C}_{\ell}\right)^{3 / 2}}\right|
$$

Let us comment on a number of features of the estimator. Firstly this statistic is global on pixel space, i.e. all the estimators are a function of all the pixel values. This means that it will be very good at identifying the scale dependence of non-Gaussianity (in the same way as the $C_{\ell}$ s are very discrimative of the scale dependence of the variance of the fluctuations); however it performs very poorly at identifying the location of any signal on the map. In this it contrasts with statistics which have been applied to the COBE data until now: the three point correlation function, the statistics of peaks and topological measures are all defined on pixel space [2]. Secondly we have assumed statistical isotropy in constructing this estimator. We know, however, that the COBE data has a number of anisotropic features which violate this assumption, in particular the presence of the galaxy (which must be removed) and the anisotropic sky coverage of the observation pattern. The only way we can truly assess the significance of the $I_{\ell}^{3}$ is by comparing them to an ensemble of $I_{\ell}^{3}$ measured on Gaussian sky maps generated with exactly the same characteristics as that of the 4 year COBE DMR data. The assumptions of the Monte Carlo must be carefully checked.

We have tested the inverse noise variance weighted, average maps of the 53A, 53B, 90A and 90B COBE-DMR channels, with monopole and dipole removed, at resolution 6 , in ecliptic pixelization. We use the extended galactic cut of [4], and [5] to remove most of the emission from the plane of the Galaxy. We apply our statistics to the DMR maps before and after correction for the plausible diffuse foreground emission outside the galactic plane as described in [6], and [7]. To estimate the $I_{\ell}^{3} \mathrm{~s}$ we set the value of the pixels within the galactic cut to 0 and the average temperature of the cut map to zero. We then integrate the map multiplied with spherical harmonics to obtain the estimates of the $a_{\ell m} \mathrm{~s}$ and apply equations 1 and 2 . We then compare the estimates to the distributions generated from Monte Carlos simulations of Gaussian maps.

Since the $I_{\ell}^{3}$ distributions are non Gaussian we generalize the $\chi^{2}$ for a set of probability functions $P_{\ell}\left(I_{\ell}^{3}\right)$ associated with observations $\left\{I_{\ell}^{3}\right\}$ by defining the following functional

$$
X^{2}=\frac{1}{N} \sum_{\ell} X_{\ell}^{2}=\frac{1}{N} \sum_{\ell}\left(-2 \log P_{\ell}\left(I_{\ell}^{3}\right)+\beta_{\ell}\right),
$$

where the constants $\beta_{\ell}$ are defined so that for each term of the sum $\left\langle X_{\ell}^{2}\right\rangle=1$. The definition reduces to the usual $X^{2}$ for Gaussian $P_{\ell}$. Again, we build a $X^{2}$ for the $C O B E-D M R$ data by means of Monte Carlo simulations. We proceed as follows. First we compute the distributions $P\left(I_{\ell}^{3}\right)$, for $\ell=2, \ldots, 18$, for a Gaussian process as measured subject to our galactic cut, and pixel noises. These $P\left(I_{\ell}^{3}\right)$ were inferred from 25000 realizations (see Fig. 1). From these distributions we then build the $X^{2}$ defined in (3), taking special care with the numerical evaluation of the constants $\beta_{\ell}$. We call this function $X_{C O B E}^{2}$. We then find its distribution $F\left(X_{C O B E}^{2}\right)$ from 


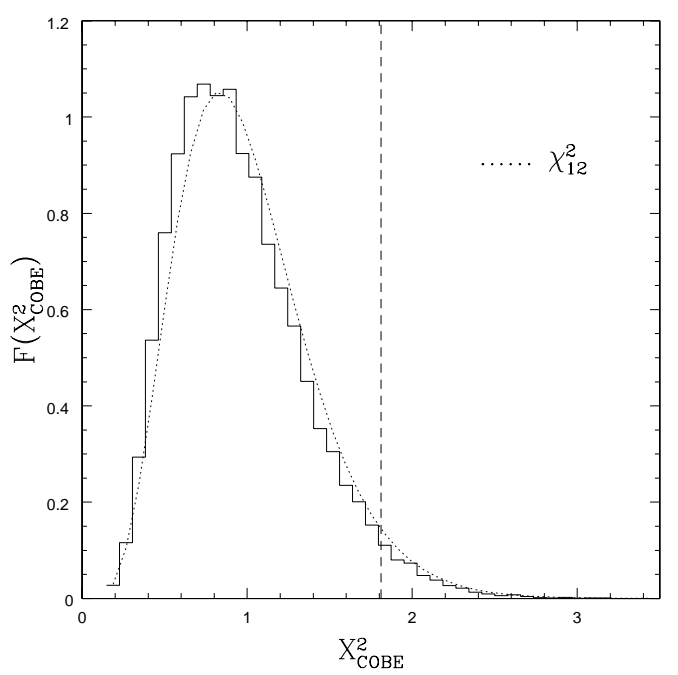

FIGURE 2. The distribution $P\left(X_{C O B E}^{2}\right)$ and a fit to it, which is a chi squared with 12 degress of freedom. The observed chi squared, with COBE, is represented as a vertical line: $X_{C O B E}^{2}=1.81$. One can compute $P\left(X_{C O B E}^{2}<1.81\right)=0.98$. Hence, it would appear that we can reject Gaussianity at the $98 \%$ confidence level.

10000 random realizations. This is very well approximated by a $\chi^{2}$ distribution with 12 degrees of freedom (Fig. 2). We then compute $X_{C O B E}^{2}$ with the actual observations and find $X_{C O B E}^{2}=1.81$. One can compute $P\left(X_{C O B E}^{2}<1.81\right)=0.98$. Hence, it would appear that we can reject Gaussianity at the $98 \%$ confidence level.

\section{IS IT A SYSTEMATIC EFFECT?}

The non-Gaussianity we are finding in the 4 year COBE DMR maps is surprising and fascinating enough that we have gone through an exhaustive test of all possible systematic effects. We summarize them in the following list:

1. Foregrounds contamination:

- Dust (using the DIRBE sky maps and also the Schlegel et al dust model)

- Synchrotron (with the Haslam template)

- Foreground corrected maps

2. Noise model:

- Anisotropic sky coverage

- Noise correlations between different pixels

- Analysis of noise templates

3. Galactic cut:

- Dependence on shape ("custom" versus constant elevation)

- Dependence on elevation

- Dependence on monopole and dipole subtraction, before or after the cut, with or with out galaxy.

4. Systematic templates

- Spurious offsets induced by the cut.

- Instrument susceptibility to the Earth magnetic field.

- Callibration errors .

- Errors due to incorrect removal of the COBE Doppler and Earth Doppler signals.

- Errors in correcting for emissions from the Earth, and eclipse effects. 

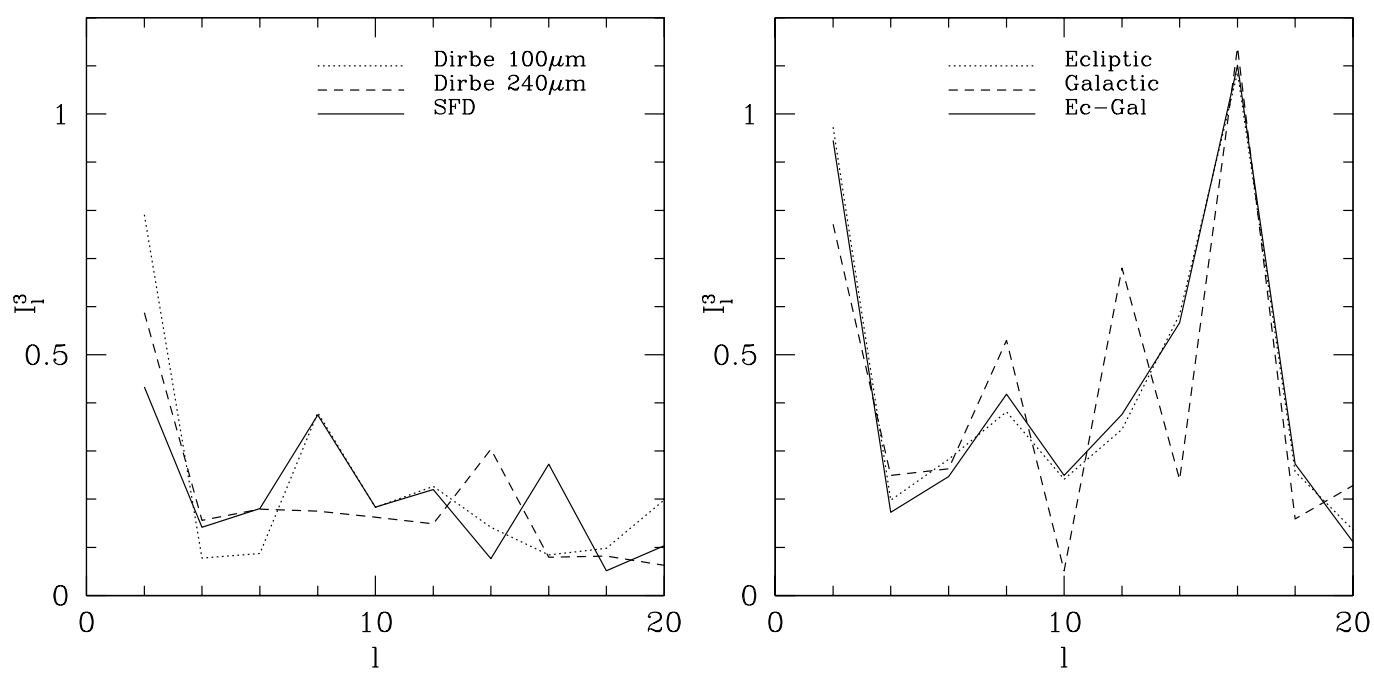

FIGURE 3. Left panel: The measured $I_{\ell}$ for dust galactic foregrounds. We have plotted values concerning the DIRBE $100 \mu \mathrm{m}$ and DIRBE $240 \mu \mathrm{m}$ as well as the Schlegel, Finkbeiner, and Davis maps. Right panel: The measured $I_{\ell}$ for DMR foreground corrected data in ecliptic coordinates, galactic coordinates, and ecliptic coordinates with galactic frame coupling.

- Artifacts due to uncertainty in the correction for the correlation created by the low-pass filter on the lock-in amplifiers (LIA) on each radiometer

- Errors due to emissions from the moon, and the planets.

5. Assumptions in Monte Carlos:

- Dependence on tilt

- Dependence on smooth versus discontinuous power spectrum

- Dependence on beam shape

- Dependence on pixelization.

Let us just highlight the foreground tests. In figure 3 (left panel) we plot the $I_{\ell}^{3} \mathrm{~s}$ estimated directly from the dust maps produced by the DIRBE $100 \mu \mathrm{m}$ and $240 \mu \mathrm{m}$ channels and from the dust template constructed in [8] using the DIRBE and IRAS maps. The feature we identify in the DMR data is not there. Furthemore, if we use the dust templates to subtract any foreground contribution (see figure 3 right panel) the value of $I_{16}^{3}$ actually increases!

We have found that none of the effects listed above change our result by much, in fact for most corrections the confidence level rises to over 99\%. We therefore claim that the non-Gaussianity we find in the COBE DMR data is not due to any of the known systematics.

\section{THE NATURE OF THE SIGNAL IN $I_{16}^{3}$}

The structure of the non-Gaussian signal is truly intriguing. It manifests itself as a spectral "spike" at $\ell=16$; it is difficult to associate such a pattern to some known or speculated source of fluctuations. If the signal is cosmological, the minimal inflationary models cannot be right. On the other hand it is not obvious that the main competitor to inflation, topological defects, could explain this type of non-Gaussianity. Topological defects are non-Gaussian, but in ways which are often more subtle than commonly thought. An interesting possibility was recently proposed by Peebles [9]. This is an isocurvature model in which the underlying fluctuations are not a Gaussian random field, but the square of a Gaussian random field. The model is based on non minimal inflation, but produces fluctuations radically different from minimal inflationary fluctuations.

A more useful exercise is to try and understand, given the characteristics of the experiment, what the power spectrum of such a non-Gaussian signal might be. The simplest thing to consider is combination of three signals. On very large scales (upto $\ell=12-14$ ) we have a Gaussian sky signal. It may be fundamentally 
Gaussian or merely a manifestation of the law of large numbers. For $\ell \geq 14$ the sky signal is non-Gaussian, and this should manifest itself all the way upto much higher $\ell$ s. However the instrument noise of the 4 Year COBE DMR data sets starts to dominate the $I_{\ell}^{3}$ s at $\ell=18-20$, where the signal to noise drops below unity. Given that the noise has been shown to be extremely well characterized by a Gaussian, the sky map will manifest itself as Gaussian for these higher $\ell$ s.

Following they publications of our result, a number of groups have reported similar results: Pando et al [11] have applied a wavelet based technique and find evidence that the non-Gaussian signal is localized in the northern hemisphere (a result we tentatively confirm [3]) while Novikov et al [12] have applied topological tests to detect non-Gaussianity in the COBE DMR data. According to skeptics, this may merely reflect a change in the psychological prior, triggered by our work. More seriously one should remember that the work performed by us and by these groups makes use of the same data set. Therefore this work provides an independent confirmation of our analysis of the DMR maps. In the very least this may mean a revision of the data analysis techniques which are currently in vogue for power spectrum estimation from CMB data sets: the underlying assumption in these Bayesian techniques is that the data set is Gaussian (greatly simplifying the estimation algorithms) [10]. However the fact that COBE data set seems to be non-Gaussian raises the question weather the current estimates of the $C_{\ell}$ s of the COBE data set are as accurate as they are claimed to be.

If the sky is truly non-Gaussian in the way we describe above, then we are at the threshold of uncovering its statistical nature with the higher resolution experiments that are coming online in particular with the

BOOMERANG LDB experiment (described elsewhere in these proceedings) and ultimately with the MAP and Planck Surveyor satellite experiments.

\section{ACKNOWLEDGMENTS}

We thank the organizers for an excellent meeting. We thank JNICT, NASA-ADP, NASA-COMBAT, NSF, RS, Starlink, TAC for support.

\section{REFERENCES}

1. Ferreira, P. G., Górski, K. M., Magueijo, J. (1998) Ap. J. Lett. 503 L1-L4.

2. Kogut, A., Banday, A. J., Bennett, C. L., Górski, K. M., Hinshaw, G., Smoot, G. F., Wright, E. L. (1996) Ap. J. 464 L29

3. Magueijo, J., Ferreira, P.G., Górski, K.M., in preparation (1999).

4. Banday, A.J., Górski, K.M., Bennett, C.L., Hinshaw, G., Kogut, A., Lineweaver, C., Smoot, G.F., and Tenorio, L. (1997) Ap.J., 475, 393

5. Bennet, C.L., Banday, A.J., Górski, K.M., Hinshaw, G., Jackson, P.D., Keegstra, P., Kogut, A., Smoot, G.F., Wilkinson, D.T., Wright, E. L. (1996) Ap. J. 464, 1

6. Kogut, A., Banday, A. J., Bennett, C. L., Górski, K. M., Hinshaw, G., Smoot, G. F., Wright, E. L. (1996) Ap. J. 464 L5

7. Górski, K. M., Hinshaw, G., Banday, A. J., Bennett, C. L., Wright, E. L., Kogut, A., Smoot, G. F., Lubin, P. (1996) Ap. J. Lett. 464, L11

8. Schlegel, D., Finkbeiner, D., Davis, M. (1998) AP. J 500, 525-553

9. Peebles, J. astro-ph/9805195 astro-ph/9805212

10. Górski, K. M., in Microwave Background Anisotropies, Proceedings of the XVIth Moriond Astrophysics Meeting, March 1996, Editions Frontieres 1997; also astro-ph/9701191

11. J. Pando, D. Valls-Gabaud, L.-. Fang, astro-ph/9810165.

12. D. Novikov, H. Feldman, S. Shandarin, astro-ph/9809238. 